\title{
Erratum to: The Effect of Loop Curvature on Coronal Loop Kink Oscillations
}

\author{
Tom Van Doorsselaere • Erwin Verwichte • \\ Jaume Terradas
}

Published online: 2 August 2014

(C) Springer Science+Business Media Dordrecht 2014

Erratum to: Space Sci. Rev. (2009) 149:299-324
DOI 10.1007/s11214-009-9530-9

Unfortunately, the credit to the author of Figs. 9, 10,11 and 12a was not given in the main text of the review. The author of these plots, who also performed the corresponding numerical calculations, was Prof. Ramon Oliver.

The online version of the original article can be found under doi:10.1007/s11214-009-9530-9.

T. Van Doorsselaere $(\varangle) \cdot$ E. Verwichte

Centre for Fusion, Space and Astrophysics, Physics Department, University of Warwick, Coventry, CV4 7AL, UK

e-mail: t.van-doorsselaere@warwick.ac.uk

J. Terradas

Centre for Plasma Astrophysics and Leuven Mathematical Modeling and Computational Science

Centre, Katholieke Universiteit Leuven, Celestijnenlaan 200B, 3001 Leuven, Belgium 